\title{
Intraabdominal Approach During Donation-After- Cardiac-Death Procurement May Offer a Simpler and Faster Way to Achieve Thoracic Aorta Cross-Clamp
}

\author{
Jay A. Graham, MD, MBA, , ${ }^{1,2}$ Gaurav Patel, MD, ${ }^{2}$ Yoshifumi Miura, MD, ${ }^{2}$ Milan Kinkhabwala, MD, ${ }^{1,2}$ Juan \\ P. Rocca, $M^{1,2}$ \\ ${ }^{1}$ Albert Einstein College of Medicine, Bronx, NY ${ }^{2}$ Montefiore-Einstein Center for Transplantation, Montefiore Medical Center, Bronx, NY
}

\section{TO THE EDITOR}

The number of donation-after-cardiac-death (DCD) procurements has continued to rise in recent years because of the unmet demand of patients on the transplant waitlist. ${ }^{1}$ However, these organs are predisposed to challenges arising from ischemic insults. Here, we describe a technique that can potentially speed the time to cross-clamp.

DCD procurement proceeds in the standard fashion after the requisite 5-minute no-touch time. A generous midline incision is made, followed by medial viscera rotation to gain access to the retroperitoneum and aorta. The aorta is emergently cannulated to flush the abdominal organs. Classically, a sternotomy is made, and the left

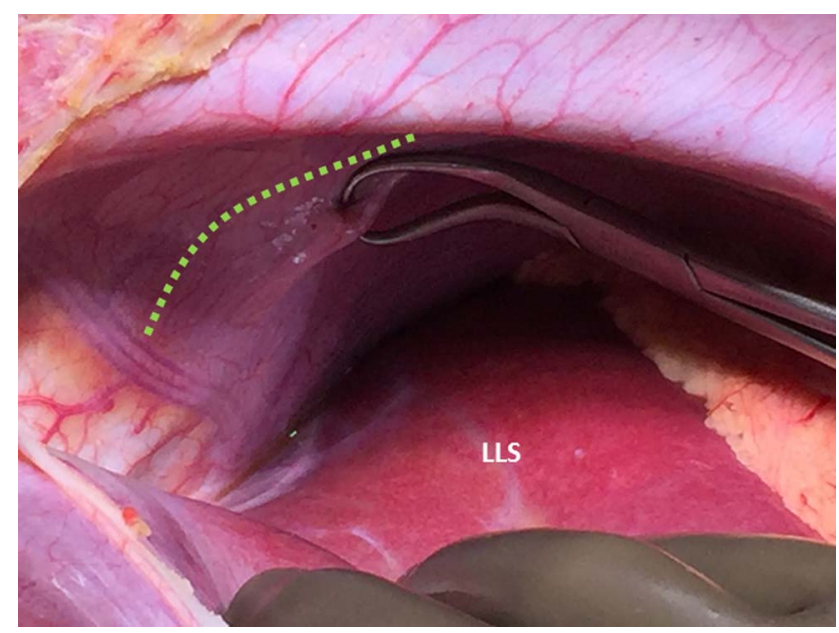

Figure. The left lateral segment (LLS) is depressed, and transection of the diaphragm (dotted line) allows for crossclamping the aorta in the chest. chest is entered through blunt dissection of the pleura with application of the aortic clamp. The donor can be vented by cutting the suprahepatic inferior vena cava (IVC) above the diaphragm, although many procuring surgeons elect to vent with a venotomy in the infrarenal IVC after insertion of the aortic cannulation.

An intraabdominal approach to clamping in the chest obviates the need to open the chest and potentially saves time. After the flush begins with insertion of the aortic cannulation, a penetrating towel clamp is used to evert the left diaphragm (Figure). The left chest is entered first, followed by entry of the pericardium. The right heart is cut to vent the donor, and the thoracic aortic clamp is applied.

This relatively simple maneuver has two potential advantages in that it is likely faster than the standard method and mitigates any potential injury to the right ureter because venting does not occur in the infrarenal IVC. Once crossclamping and venting have occurred, the DCD procurement proceeds in the standard fashion. A sternotomy is made to assess the intrathoracic organs, and the suprahepatic IVC can be cut in a manner similar to the conventional approach.

The mean time to cross-clamping for 10 DCD procurements done in this fashion was 2.9 minutes.

While this maneuver has few contraindications, the approach obviously cannot be used if lung procurement is planned. Also, if the donor is a medical examiner case, the procuring surgeon must understand the limitations. Although right heart ventriculotomy is a preferred maneuver for expedient venting, the medical examiner may have some reservations.

\section{REFERENCE}

1. Kim WR, Lake JR, Smith JM, et al. OPTN/SRTR 2016 Annual Data Report: Liver. Am J Transplant. 2018 Jan;18 Suppl 1:172-253. doi: 10.1111/ajt.14559. 